\title{
Adaptive Control of Vehicle Active Suspension Based on Neural Network Optimization
}

\author{
Runqi Qiu ${ }^{1 *}$ \\ ${ }^{1}$ School of International Education, Wuhan University of Technology, Hubei, Wuhan, 430070, China
}

\begin{abstract}
An adaptive proportional-integral-derivative (PID) control method based on radial basis function neural network optimization (RBF-PID) is designed for a four-degree-of-freedom active suspension model of a $1 / 2$ vehicle. By building a simulation model of the suspension in MATLAB/Simulink, a C-level road white noise random excitation signal is used as the road input, and the front and rear body vertical accelerations are simulated as the feedback of the control loop, respectively. The simulation results show that the proposed RBF-PID control strategy can effectively suppress the front and rear body acceleration and effectively reduce the centroid vertical acceleration, and improve the performance by about $10.3 \%$ compared with the traditional PID control suspension and about 31.2\% compared with the passive suspension, but the control effect improvement for the angular acceleration of body pitch angle is not obvious.
\end{abstract}

\section{Introduction}

Nowadays, with the rapid development of the automobile industry, people's requirements for car performance are also increasing, and the comfort and stability of the car have become the primary consideration when people buy a car. The comfort and stability are closely related to the performance of automobile suspension. In the process of vehicle driving, the car's suspension will actively absorb the vibration brought by the road to enhance the comfort of passengers and the stability of the owner's operation.

The traditional passive suspension of the automobile is composed of a shock absorber and spring. Once the damping coefficient of the damper and the stiffness of the spring are determined, it is difficult to adjust dynamically according to the working conditions and cannot react differently under different road conditions. However, the road conditions encountered by the car are changing during the driving process, so the suspension has to be adjusted accordingly to fit the actual driving conditions of the car. Active suspension is a good solution to the problem of non-adjustable passive suspension parameters. The ECU of the car understands the body motion condition through the acceleration sensors all over the body and adaptively adjusts the suspension performance so that the suspension system can be continuously limited in an optimal damping state. For this reason, active suspension systems have become a hot topic in suspension system research today. Based on the passive suspension, the active suspension adds an actuator that dynamically adjusts the actuation force to provide a main force inverse to the body impact load at any time to counteract the impact from the road, thus achieving the damping effect.

Since the middle and late 19th century, experts and scholars have done a lot of research on control strategies, while putting forward various control theories. In recent years, many scholars have started to apply neural network control systems to suspension control and achieved good control result with the help of their strong learning ability and high robustness.

However, most of the studies on active suspension control strategy are based on the two-degree-of-freedom model of 1/4 car, ignoring the influence of front and rear suspension vertical motion on the body pitch angle. Therefore, in this paper, an RBF-PID controller is designed based on a four-degree-of-freedom model of a $1 / 2 \mathrm{car}$, and the front and rear body vertical accelerations are used as the feedback of the controller to adjust the active suspension dynamics, respectively. The simulation results show that the method improves the suspension performance, but there is still some room for improvement.

\section{System Description}

\subsection{System Description}

Since the vehicle is a complicated vibration model, it is essential to simplify the system based on the analysed problem. Although the 2-DOF quarter vehicle model has a simple structure, it can only indicate the vertical acceleration and velocity. The 7-DOF full vehicle model can characterize the vertical, roll as well as pitch motions, but it is too sophisticated for the designing of the controller. Considering the difficulty of system modelling, controller design as well as simulation, this paper will adopt the 4-DOF half vehicle model as the plant.

In these research, four assumptions have been established on the suspension system:

*Corresponding author: qiurunqi@whut.edu.cn 
- The suspension can be simplified to a spring-massdamper system.

- The connection between the wheel and the axle and the connection between the body and the seat can be simplified to a rigid connection.

- The tire can be regarded as a linear spring without damping and assured to contact the road when driving.

- The vehicle makes a uniform linear motion in the longitudinal direction.

The simplified half-car model is shown in Fig.1, in which sprung mass $m_{2 f}$ and $m_{2 r}$ represent the mass of vehicle body $m_{2}$ distributed on the front axle and the rear axle, respectively. The equivalent stiffness coefficient of the tire is $k_{t f}$ and $k_{t r} . c_{f}$ and $c_{r}$ represent the damping ratio of the linear damper and the stiffness of the linear spring is $k_{f}$ and $k_{r}$. The vehicle has a moment of inertia of $I_{y}$ and pitch angle of $\varphi$. The actuation force $u_{f}$ and $u_{r}$ is acting between the car body and the tire. Front axle and rear axle distances with the centre of gravity are $a$ and $b$. $Z_{2 f}$ and $Z_{2 r}$ stands for the vertical displacement of the sprung mass, while $Z_{1 f}$ and $Z_{1 r}$ represent that of the unsprung mass.

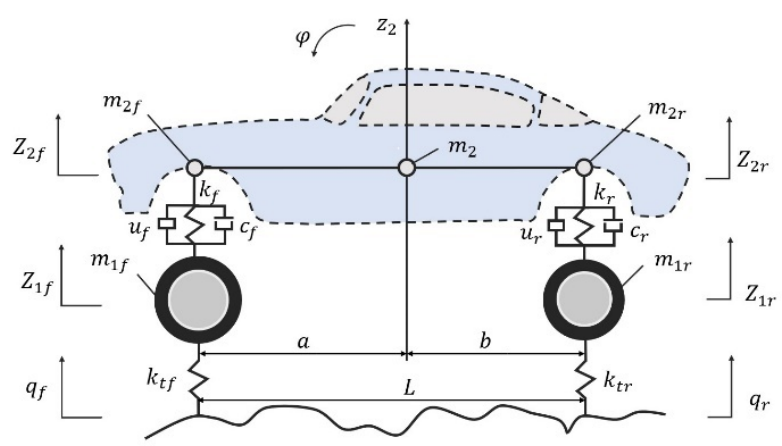

Fig. 1. Half-Vehicle Active Suspension Model

Based on the free body diagram and Newton's second law, the following system dynamic differential equation can be obtained:

$$
\begin{gathered}
m_{2} \ddot{Z}_{2}+k_{f}\left(Z_{2 f}-Z_{1 f}\right)+c_{f}\left(\dot{Z}_{2 f}-\dot{Z}_{1 f}\right)+u_{f} \\
+k_{r}\left(Z_{2 r}-Z_{1 r}\right) \\
+c_{r}\left(\dot{Z}_{2 r}-\dot{Z}_{1 r}\right)+u_{r}=0 \\
m_{1 f} \ddot{Z}_{1 f}-k_{f}\left(Z_{2 f}-Z_{1 f}\right)-c_{f}\left(\dot{Z}_{2 f}-\dot{Z}_{1 f}\right) \\
-u_{f}+k_{t f}\left(Z_{1 f}-q_{f}\right)=0 \\
m_{1 r} \ddot{Z}_{1 r}-k_{r}\left(Z_{2 r}-Z_{1 r}\right)-c_{r}\left(\dot{Z}_{2 r}-\dot{Z}_{1 r}\right) \\
-u_{r}+k_{t r}\left(Z_{1 r}-q_{r}\right) \\
=0
\end{gathered}
$$

$$
\begin{aligned}
I_{y} \ddot{\varphi}-a\left[k _ { f } \left(Z_{2 f}\right.\right. & \left.\left.-Z_{1 f}\right)+c_{f}\left(\dot{Z}_{2 f}-\dot{Z}_{1 f}\right)+u_{f}\right] \\
& +b\left[k_{r}\left(Z_{2 r}-Z_{1 r}\right)\right. \\
& \left.+c_{r}\left(\dot{Z}_{2 r}-\dot{Z}_{1 r}\right)+u_{r}\right]=0
\end{aligned}
$$

When the pitch angle $\varphi$ of the body is small, $\tan \varphi$ is approximately equal to $\varphi$. Thus, the vertical displacement of the sprung mass of the front and rear suspension can be expressed as follows:

$$
\begin{aligned}
& Z_{2 f} \approx Z_{2}-Z_{1 f}-a \varphi \\
& Z_{2 r} \approx Z_{2}-Z_{1 r}-a \varphi
\end{aligned}
$$

When the actuation force $u_{f}$ and $u_{r}$ are both zero, the active suspension system becomes a passive suspension system.

The parameters used for the half-vehicle suspension model are presented in Table 1.

Table 1. System Parameters

\begin{tabular}{|c|c|c|}
\hline Parameters & Value & Unit \\
\hline$m_{2}$ & 975.37 & $\mathrm{~kg}$ \\
\hline$m_{1 f}$ & 98 & $\mathrm{~kg}$ \\
\hline$m_{1 r}$ & 98 & $\mathrm{~kg} \cdot \mathrm{m}^{2}$ \\
\hline$I_{y}$ & 1674 & $\mathrm{~kg} \cdot \mathrm{m}^{2}$ \\
\hline$k_{f}$ & 45.48 & $\mathrm{kN} / \mathrm{m}$ \\
\hline$k_{r}$ & 52.59 & $\mathrm{kN} / \mathrm{m}$ \\
\hline$k_{t f}$ & 604.69 & $\mathrm{kN} / \mathrm{m}$ \\
\hline$k_{t r}$ & 985.97 & $\mathrm{kN} / \mathrm{m}$ \\
\hline$c_{f}$ & 2546.5 & $\mathrm{~N} \cdot \mathrm{s} / \mathrm{m}$ \\
\hline$c_{r}$ & 2840.6 & $\mathrm{~N} \cdot \mathrm{s} / \mathrm{m}$ \\
\hline$a$ & 1.1135 & $\mathrm{~m}$ \\
\hline$b$ & 1.5415 & $\mathrm{~m}$ \\
\hline $\mathrm{L}$ & 2.655 & $\mathrm{~m}$ \\
\hline
\end{tabular}

\subsection{Road Excitation Model}

Based on the free body diagram and Newton's second law, the following system dynamic differential equation can be obtained:

$$
\begin{aligned}
& q_{f}=2 \pi n \sqrt{G_{0}\left(n_{0}\right) u} \int w\left(t_{1}\right) \mathrm{d} t \\
& q_{r}=2 \pi n \sqrt{G_{0}\left(n_{0}\right) u} \int w\left(t_{2}\right) \mathrm{d} t
\end{aligned}
$$

The vertical displacement of the $C$-level road profile is used as the input signal to the suspension system. The road profile roughness coefficient $G_{0}\left(n_{0}\right)=256 \times$ $10^{-6} \mathrm{~m}^{3} . w(t)$ is the uniformly distributed white noise. Assuming that the driving speed $v=20 \mathrm{~m} / \mathrm{s}$ and the rear wheel input have a 0.1 -second delay compared with the front wheel. Let the spatial reference frequency of the road excitation $n_{0}=0.1 \mathrm{~m}^{-1}$ and set the simulation time to $10 \mathrm{~s}$, the road input response of the front and rear wheels can be obtained, as shown in Fig.2.

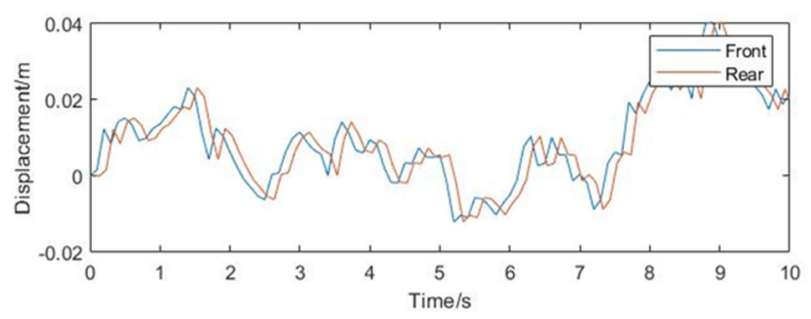

Fig. 2. Road Excitation of Front and Rear Wheel 


\section{Controller Design}

Conventional PID controllers rely heavily on precise mathematical models of the controlled objects, which is considered to be of low efficiency when applied to suspension systems. In contrast, neural networks have the superior nonlinear fitting ability and are suitable for vehicle suspension, a complex controlled object that is difficult to model accurately. Therefore, this paper improves the traditional PID controller based on neural network and selects the RBF network to design the RBFPID controller combining RBF and PID to achieve the adaptive adjustment of controller parameters as well as improving the control accuracy.

\subsection{RBF Network}

RBF neural network is a three-layer feedforward network composed of an input layer, a hidden layer, and an output layer. Since the mapping of the input to the output is nonlinear and the mapping from the hidden layer to the output layer is linear, the local minimum problem of the BP neural network with the same feedforward characteristic can be avoided, and the learning speed can be greatly improved.

The topology of the RBF neural network based on Gaussian basis functions is shown in Fig.3. In this network, the input vector of the network is $X=$ $\left[x_{1}, x_{2}, \cdots, x_{m}\right]^{T}$ and the radial basis vector of the hidden layer is $H=\left[h_{1}, h_{2}, \cdots, h_{j}, \cdots, h_{m}\right]^{T}$, where $h_{j}$ is the Gaussian basis function.

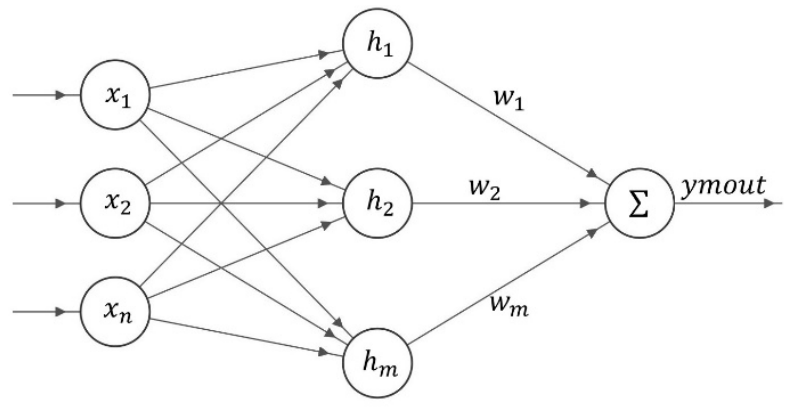

Fig. 3. RBF Neural Network

$$
h_{j}=\exp \left(-\frac{\left\|\mathrm{X}-\mathrm{C}_{\mathrm{j}}\right\|^{2}}{2 \mathrm{~b}_{\mathrm{j}}^{2}}\right), \mathrm{j}=1,2,3, \cdots, \mathrm{m}
$$

In the equation, $C_{j}$ is the centre vector of the $j$ th node in the network, $C_{j}=\left[c_{j 1}, c_{j 2}, \cdots, c_{j i}, \cdots, c_{j m}\right]^{T}$; Let $B=$ $\left[b_{1}, b_{2}, \cdots, b_{j}, \cdots, b_{m}\right]^{T}, b_{j}$ being the base width parameter of the hidden layer node $j$ and satisfies $b_{j}>0$.

Assuming that the weight vector of the network is $W=\left[w_{1}, w_{2}, \cdots, w_{j}, w_{m}\right]^{T}$, the output of the network can be obtained:

$$
y_{m}(k)=\sum_{j=1}^{m} w_{j} h_{j}
$$

Let the theoretical output of the discriminative system at time $k$ is yout $(k)$, and the output of this identification network is $\operatorname{ymout}(k)$, then the performance index function of the network is:

$$
J=\frac{1}{2}\left(y(k)-y_{m}(k)\right)
$$

Let $\alpha$ be the moment factor and $\eta$ be the learning rate. According to the gradient descent method, the iterative algorithm of weight factor $w$, the node centre vector $c$ and the node base width parameter $b$ can be obtained as follows:

$$
\begin{gathered}
w_{j}(k)=w_{j}(k-1)+\eta\left(y(k)-y_{m}(k)\right) h_{j} \\
+\alpha\left(w_{j}(k-1)-w_{j}(k\right. \\
-2))
\end{gathered}
$$

$$
\begin{gathered}
\Delta b_{j}=\left(y(k)-y_{m}(k)\right) w_{j} h_{j} \frac{|| X-\left.C_{j}\right|^{2}}{b_{j}^{3}} \\
b_{j}(k)==b_{j}(k-1)+\eta \Delta b_{j}+\alpha\left(b_{j}(k-1)\right. \\
\left.-\left(b_{j}(k-2)\right)\right) \\
\Delta c_{j i}=\left(y(k)-y_{m}(k)\right) w_{j} \frac{x_{j}-c_{j i}}{b_{j}^{2}} \\
c_{j i}(k)=c_{j i}(k-1)+\eta \Delta c_{j i}+\alpha\left(c_{j 1}(k-1)\right. \\
\left.-c_{j 1}(k-2)\right)
\end{gathered}
$$

\subsection{PID Parameters Tuning Based on RBF}

RBF-PID uses the incremental PID control method:

$$
\begin{gathered}
\Delta u(k)=K_{P}(e(k)-e(k-1))+K_{I} e(k) \\
+K_{D}[e(k)-2 e(k-1) \\
+e(k-2)] \\
u(k)=u(k)+\Delta u(k)
\end{gathered}
$$

Where $e(k)$ is the control error, $e(k)=\operatorname{rin}(k)-$ $y(k)$. For the active suspension, $\operatorname{rin}(k)$ represents the desired front and rear vertical acceleration of the vehicle body.

The Three inputs to the controller are:

$$
\begin{gathered}
x c(1)=e(k)-e(k-1) \\
x c(2)=e(k) \\
x c(3)=e(k)-2 e(k-1)+e(k-2)
\end{gathered}
$$

The Gain parameters $K_{P}, K_{I}$ and $K_{D}$ of the PID are adjusted using the gradient descent method.

$$
\begin{aligned}
\Delta K_{P}=\eta_{P} \frac{\partial E}{\partial K_{P}}= & -\eta_{P} \frac{\partial E}{y} \frac{\partial y}{\partial u} \frac{\partial u}{\partial K_{P}} \\
& =\eta_{P} e(k) \frac{\partial y}{\partial u} x c(1) \\
\Delta K_{I}=\eta_{I} \frac{\partial E}{\partial K_{I}}= & -\eta_{I} \frac{\partial E}{y} \frac{\partial y}{\partial u} \frac{\partial u}{\partial K_{I}} \\
& =\eta_{I} e(k) \frac{\partial y}{\partial u} x c(1)
\end{aligned}
$$




$$
\begin{aligned}
\Delta K_{D}=\eta_{D} \frac{\partial E}{\partial K_{D}}= & -\eta_{D} \frac{\partial E}{y} \frac{\partial y}{\partial u} \frac{\partial u}{\partial K_{D}} \\
& =\eta_{D} e(k) \frac{\partial y}{\partial u} x c(1)
\end{aligned}
$$

Where $\partial y / \partial u$ is the Jacobian information of the controlled object, that is, the sensitivity of the output to the input, which can be obtained by the recognition of the neural network as follows:

$$
\frac{\partial y(k)}{\partial u(k)} \approx \frac{\partial y_{m}(k)}{\partial u(k)}=\sum_{j=1}^{m} w_{j} h_{j} \frac{C_{j 1}-x_{1}}{b_{j}^{2}}
$$

Based on the above mathematical principles, the RBFPID controller can be established in MATLAB/Simulink as shown in Fig.4.

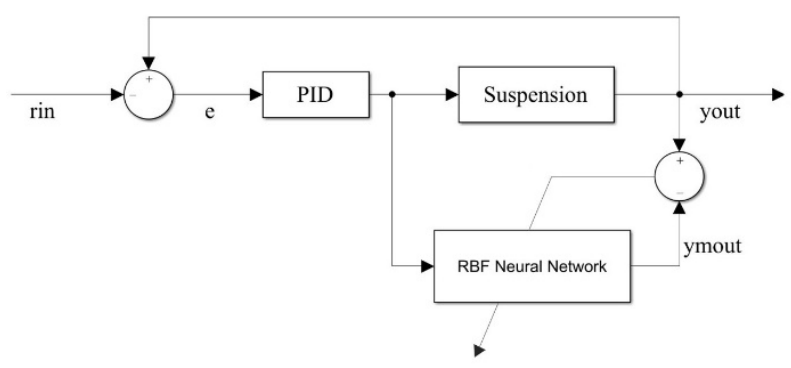

Fig. 4. RBF-PID Controller

\section{Data Analysis and Comparisons}

\subsection{System Simulation}

The learning rate $\eta$ and the moment factor $\alpha$ of the RBF neural network are real numbers that greater than 0 and less than 1 . Take $\eta=0.25$ and $\alpha=0.05$ and set the number of hidden layer nodes to 6 based on experience. The gain parameters of the PID controller are set to $K_{p}=$ $55, K_{I}=14, K_{D}=1.5$. The adaptive adjustment of the PID gain parameters optimized by the RBF neural network with the change of the road input is shown in Fig.5 and Fig.6.
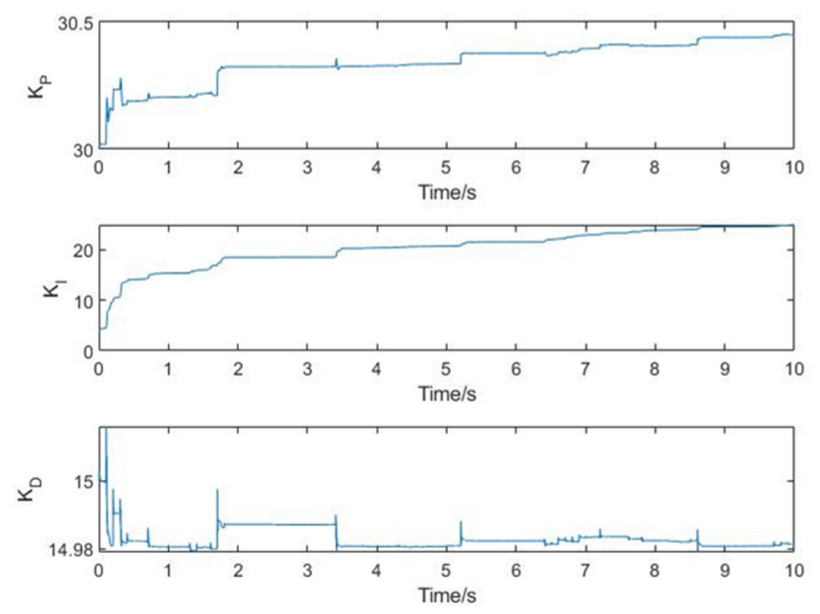

Fig. 5. Front Controller Parameter Changes
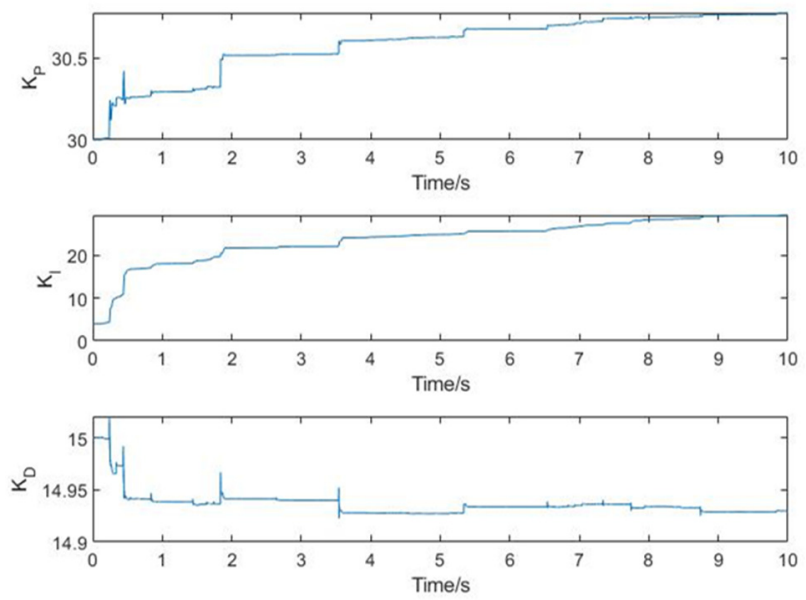

Fig. 6. Rear Controller Parameter Changes

\subsection{Controller Performance Comparison}

In the simulation experiment, this paper focuses on the vehicle body centroid vertical acceleration and pitch angle acceleration. Among them, the centre-of-mass acceleration characterizes the intensity of body vibration when driving on uneven road, and this index mainly affects the ride comfort of the vehicle; the pitch angle acceleration characterizes the body nodding or lifting caused by the vehicle during emergency braking or rapid acceleration, and this situation will cause the driver and passenger to lean back and forward, which affects the ride comfort.

The vertical acceleration of the front and rear bodies was simulated as the feedback of the closed-loop control, and the output results of the passive suspension were compared with the output results of the RBF-PIDcontrolled suspension and PID-controlled suspension as well as passive suspensions under the same road input in the same coordinate system, and the simulation results are shown in Fig. 7 to Fig. 10.

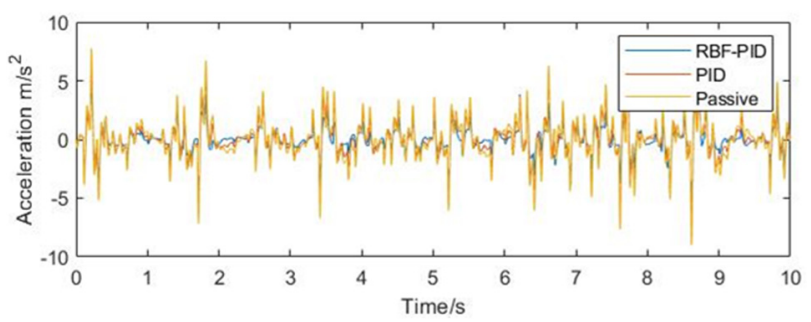

Fig. 7. Front Body Vertical Acceleration

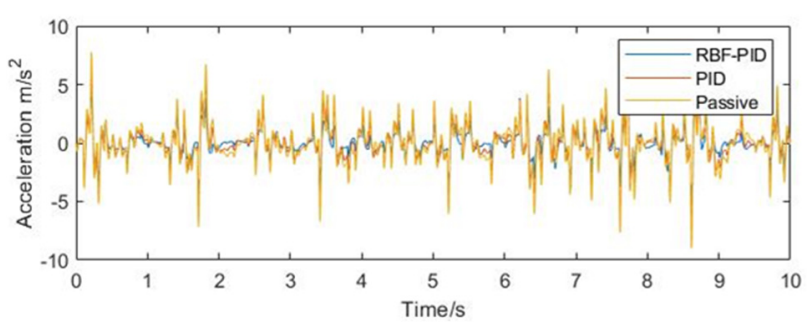

Fig. 8. Rear Body Vertical Acceleration 


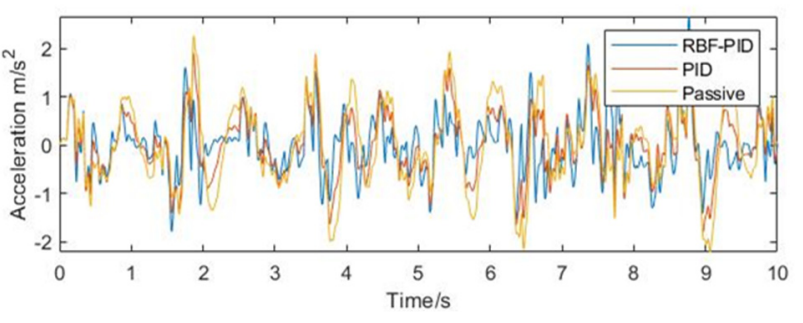

Fig. 9. Centroid Acceleration

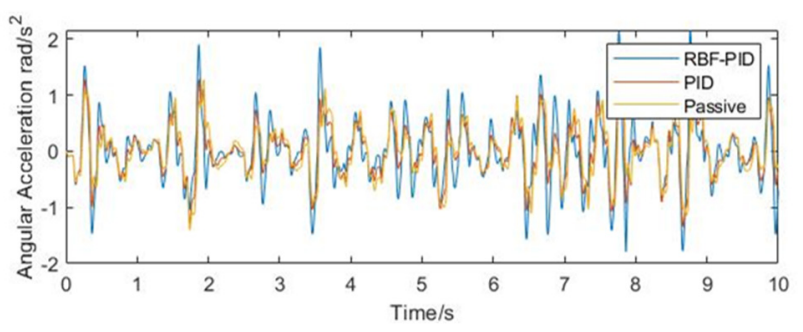

Fig. 10. Pitch Angle Acceleration

Calculating the root mean square values of the three and analysing the dispersion of the data, the results shown in Table 2 can be obtained.

Table 2. Simulation Results

\begin{tabular}{|c|c|c|c|c|}
\hline & $\ddot{Z}_{2 f}$ & $\ddot{Z}_{2 r}$ & $\ddot{Z}_{2}$ & $\ddot{\boldsymbol{\varphi}}$ \\
\hline Passive & 1.7728 & 2.2876 & 0.8566 & 0.4672 \\
\hline PID & 1.4530 & 1.8783 & 0.6576 & 0.4619 \\
\hline RBF-PID & 1.1122 & 1.2619 & 0.5896 & 0.5683 \\
\hline
\end{tabular}

As can be seen from Table 2, compared with the passive suspension and PID-controlled suspension, the controller designed in the thesis has a very obvious improvement in reducing the vertical vibration velocity under random road input, which effectively improves the ride comfort and handling stability performance of the vehicle. However, the pitch angle acceleration improvement is not obvious, probably because the coupling relationship between the two is not taken into account when establishing the control loops for the front and rear suspensions separately.

\section{Conclusion}

In this paper, an RBF-PID-based active suspension with adaptively tuned parameters is designed. The parameters of the PID controller are adaptively adjusted online by RBF-PID to achieve a stable output of the system. The active suspension is modelled in MATLAB/Simulink, and the body vertical acceleration and body angular acceleration of PID, RBF-PID-controlled suspension, and conventional passively controlled suspension are compared under C-level random road input. The simulation results show that the RBF-PID outperforms the PID controller.

Considering that the controller designed in this study uses two separate single loops to control the front and rear suspensions respectively, the coupling relationship between the two is neglected. Although the controller designed in the thesis has good results for the acceleration of the front and rear ends of the vehicle as well as the acceleration of the centre of mass, the control of the angular acceleration of the pitch angle of the vehicle is not satisfactory. The next research will try to use the MPC algorithm to solve the coupling relationship between the front and rear suspensions, and if the effect is significant, then we will start the test based on the real car.

\section{References}

1. Zhang, M.G., Li, W.H., Liu, M.Q. (2005) Adaptive PID Control Strategy Based on RBF Neural Network Identification. In: 2005 International Conference on Neural Networks and Brain, Beijing.pp. 1854-1857.

2. Ikenaga, S., Lewis, F.S., Davis, L. (2000) Active suspension control of ground vehicle based on a fullvehicle model. In: 2000 American Control Conference, Chicago.pp. 4019-4024.

3. Thompson, A.G., Davis, B.R. (1992) Optimal Active Suspension Design Using a Frequency-shaping PID Filter. Vehicle System Dynamics, 1: 19-37

4. Khan, L., Qamar, S., Khan U. (2016) Adaptive PID control scheme for full car suspension control. Journal of the Chinese Institute of Engineers, 2: 169185.

5. Zhang, M.G., Wang, X.G., L, M.Q. (2005) Adaptive PID control based on RBF neural network identification. In: 17th IEEE International Conference on Tools with Artificial Intelligence. Hong Kong. pp. 3 pp.-683.

6. Lin, J., Lian, R. (2011) Intelligent Control of Active Suspension Systems. IEEE Transactions on Industrial Electronics, 2 :618-6228. 